\title{
Nicorandil Induced Colonic Ulcer: A Late Complication?
}

\author{
Ravindra S Date ${ }^{\mathrm{a}, \mathrm{b}}$, Olatunde S. Owa ${ }^{\mathrm{a}}$, Muntzer M Mughal ${ }^{\mathrm{a}}$
}

\begin{abstract}
Nicorandil is a third line treatment for ion heart disease. Oral and anal ulceration are recogpical ale effects of Nicorandil occurring within fewhring of 6 mmencing the treatment. Colonic ulcers are rare. W puesent a case of an 82-year-old woman who developed colonic ulcers 8 years after commencing on Nicorandil. The ulcers healed within 6 months of discontinuing the treatment. The case may suggest colonic ulceration as long term effect of the drug.
\end{abstract}

Keywords: Nicorandil; Colon; Ulcer

\section{Introduction}

Nicorandil is a third line treatment for ischemic heart disease. Oral and anal ulceration are recognized side effects of Nicorandil occurring within few months of commencing the treatment. Colonic ulcers are rare. We present a case of an 82-year-old woman who developed colonic ulcers 8 years after commencing on Nicorandil. The ulcers healed within 6 months of discontinuing the treatment. The case may suggest colonic ulceration as long term effect of the drug.

\section{Case Report}

An 82-year-old lady presented with abdominal pain and constipation. She had colonoscopy which revealed a 2 x 2 $\mathrm{cm}$ polyp at $22 \mathrm{~cm}$. This was excised. Histology showed a tubulovillous adenoma with mild dysplasia. She had 2 fur-

Manuscript accepted for publication April 21, 2010

${ }^{a}$ Department of Gastrointestinal Surgery, Lancashire Teaching Hospitals NHS, Foundation Trust, Preston Road, Chorley, Lancashire, PR7 1PP, UK

${ }^{\mathrm{b}}$ Corresponding author, Email: ravidate@hotmail.com

doi:10.4021/gr2010.04.191w

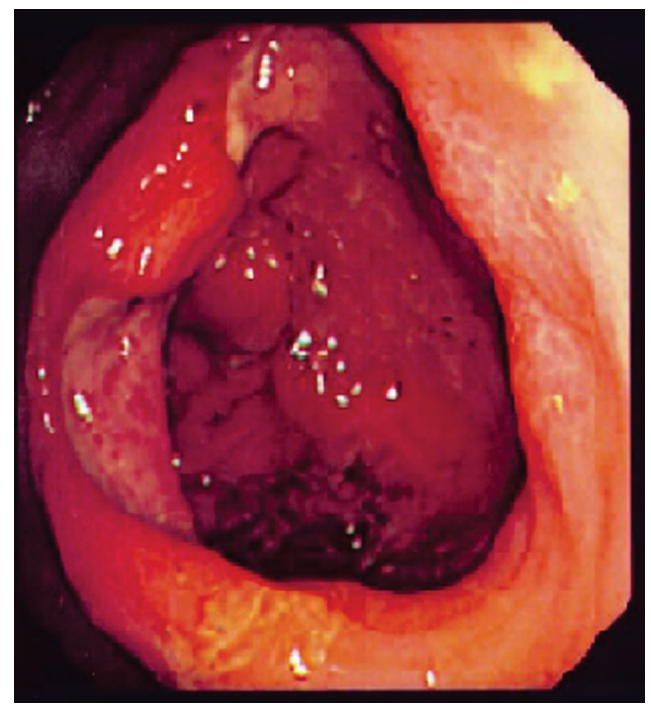

Figure 1. Ulceration in transverse colon noted 8 years after commencing Nicorandil.

ther "follow-up" colonoscopies at 2 yearly intervals. These were normal. A further colonoscopy 3 years later showed circumferential ulcer at the junction of the proximal and

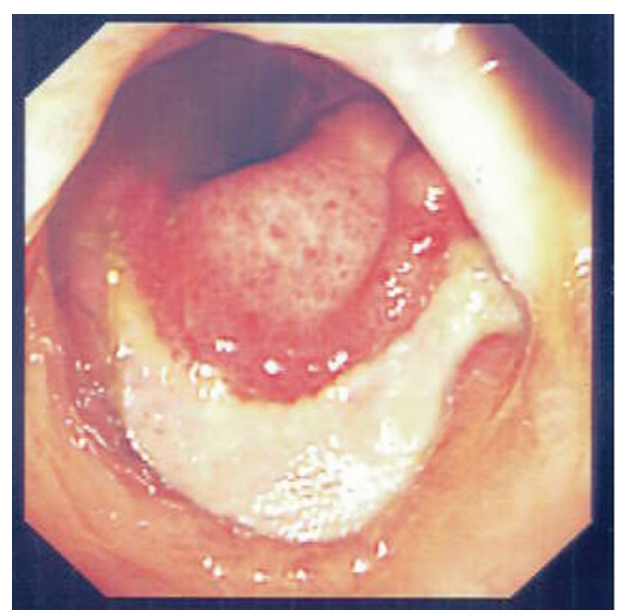

Figure 2. Persistent ulceration 2 years later. 


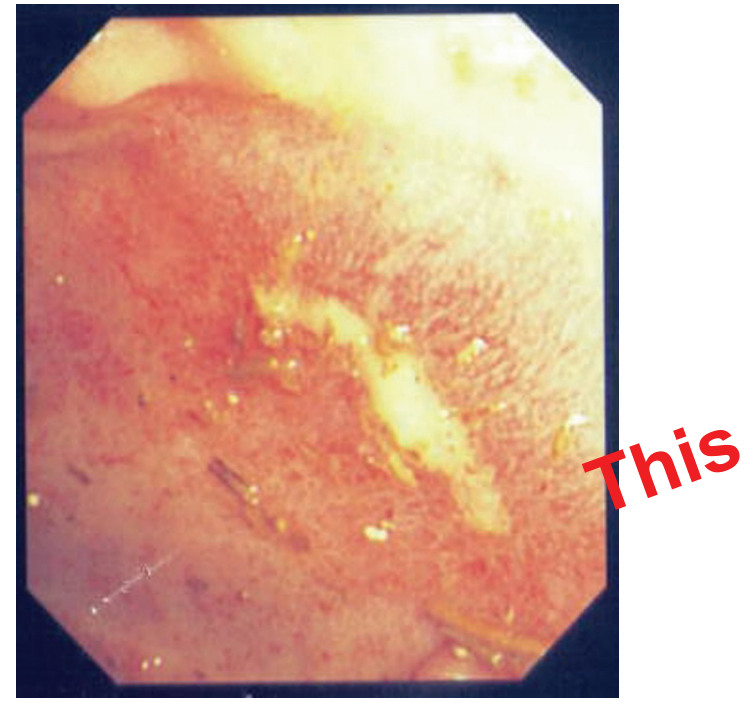

Figure 3. Healed ulcer 6 months after stopping Nicorandil.

middle third of transverse colon (Fig. 1). Histology showed non-specific inflammatory changes; there was no evidence of granulomata, dysplasia or neoplasia. Persistent ulceration was noted on 2 further colonoscopies at one year intervals (Fig. 2). Biopsies were benign on both the occasions. The ulceration was thought to be secondary to mucosal prolapse. At this stage after going through her detailed history it was noted that she suffered from ischemic heart disease and had been on nicorandil $30 \mathrm{mg}$ twice a day for the last 13 years. After consultation with the cardiologist, Nicorandil was discontinued and dose of Isosorbide nitrate was increased. Further colonoscopy 6 months later showed healing of an ulcer (Fig. 3).

\section{Discussion}

Nicorandil, a potassium activator is a third line drug in the treatment of severe ischaemic heart disease [4]. It acts by relaxing the smooth muscle of the blood vessels, causing both arterial and venous vasodilatation. Its use has been associated with oral and anal ulcerations $[6,7]$, but ulcers at other sites in gastrointestinal tract have also been described $[8,9]$.

Pathogenesis of these ulcers is not known, but they appear to be related to the dose of Nicorandil and resolve upon withdrawal of the drug with median healing time of 12 weeks. Other proposed mechanisms are vascular steal phenomenon and direct local effect of either Nicorandil itself or its metabolite [5].

Most of the oral ulcers develop within few months of commencing the Nicorandil [10]. Our patient though on a high dose of Nicorandil, developed ulcers in the colon 8 years after commencing treatment suggesting that this may be a long term effect of the drug. Her 3 colonoscopies during these 8 years did not show any evidence of ulceration. Healing of ulcer on discontinuing the medication confirmed the etiological role of Nicorndil.

Nicorandil should be considered in the dif efentral diagnosis of idiopathic gastrointestinal id fron to avoid unnecessary surgical intervention ithicians prescribing the drug should be awa of side effect. This drug is used for ischemis disease and patients using this are also more lo ly to be on anticoagulants which increase the risk Theeding from ulcers. Surgeons treating the patients with per rectal bleeding should consider discontinuing Nicorandil along with anticoagulation in appropriate circumstances.

Most of the ulcers develop within few months of commencing the Nicorandil [10]. Our patient developed ulcer in colon 8 years after commencing the treatment suggesting that this may be a long term effect of the drug.

\section{Competing Interest}

None to declare.

\section{References}

1. Abdelrazeq AS, Owen C, Smith L, McAdam JG, Pearson HJ, Leveson SH. Nicorandil-associated para-stomal ulceration: Case series. Eur J Gastroenterol Hepatol 2006;18(12):1293-1295.

2. Egred M. Nicorandil-associated ulcerations. Eur J Gastroenterol Hepatol 2007;19(5):395-398.

3. Brown R, Lee A, Welfare M. Nicorandil-induced colonic ulceration. Heart 2008;94(5):678.

4. Titi MA, Seow C, Molloy RG. Nicorandil-Induced Colonic Ulceration: A New Cause of Colonic Ulceration. Report of Four Cases. Dis Colon Rectum 2008.

5. Toquero L, Briggs CD, Bassuini MM, Rochester JR. Anal ulceration associated with Nicorandil: case series and review of the literature. Colorectal Dis 2006;8(8):717720.

6. Watson A, Suttie S, Fraser A, O'Kelly T, Loudon M. Nicorandil associated anal ulceration. Colorectal Dis 2004;6(5):330-331.

7. Webster K, Godbold P. Nicorandil induced oral ulceration. Br Dent J 2005;198(10):619-621.

8. Egred M, Andron M, Morrison WL. Nicorandil may be associated with gastrointestinal ulceration. Bmj 2006;332(7546):889.

9. King PM, Suttie SA, Jansen JO, Watson AJ. Perforation of the terminal ileum: a possible complication of nicorandil therapy. Surgeon 2004;2(1):56-57.

10. Gupta A, Morris G. Major aphthous ulcers induced by nicorandil. Age Ageing 2000;29(4):372-373. 